\title{
Unicité et minimalité des solutions d'une équation de Ginzburg-Landau
}

par

\author{
Gilles CARBOU
}

Centre de Mathématiques

et de leurs Applications (URA 1611),

École Normale Supérieure de Cachan,

61, avenue du Président Wilson, 94235 Cachan Cedex

RÉSUME. - On étudie les solutions de l'équation $-\Delta u+u\left(u^{2}-1\right)=0$, où $u$ est élément de $H_{l o c}^{l}\left(\mathbb{R}^{n}\right)$. On montre que la solution $f\left(x_{1}, \ldots, x_{n}\right)=$ th $\left(\frac{x_{1}}{\sqrt{2}}\right)$ minimise l'énergie parmi les fonctions tendant vers -1 lorsque $x_{1}$ tend vers $-\infty$ et vers +1 lorsque $x_{1}$ tend vers $+\infty$. De plus, on prouve que toute solution de l'équation qui tend vers 1 lorsque $|x|$ tend vers $+\infty$ est constante égale à 1 sur $\mathbb{R}^{n}$.

ABStraCt. - We study the solutions of the equation $-\Delta u+u\left(u^{2}-1\right)=0$ where $u$ is in $H_{l o c}^{1}\left(\mathbb{R}^{n}\right)$. We prove that the map $f\left(x_{1}, \ldots, x_{n}\right)=\operatorname{th}\left(\frac{x_{1}}{\sqrt{2}}\right)$ is a minimizing solution in the class of functions which converge to -1 when $x_{1}$ goes to $-\infty$ and to +1 when $x_{1}$ goes to $+\infty$. Furthermore we show that any solution of the equation which converges to 1 when $|x|$ goes to $+\infty$ is constant equal to 1 on $\mathbb{R}^{n}$. 


\section{INTRODUCTION}

On considère l'équation sur $\mathbb{R}^{n}$ :

$$
-\Delta u+u\left(u^{2}-1\right)=0
$$

où $u$ est élément de $H_{\text {loc }}^{1}\left(\mathbb{R}^{n}\right) \cap L^{\infty}\left(\mathbb{R}^{n}\right)$, où

$$
H_{\text {loc }}^{1}\left(\mathbb{R}^{n}\right)=\left\{u \text {, pour tout ouvert borné } \Omega \text { de } \mathbb{R}^{n}, u \in H^{1}(\Omega)\right\} \text {. }
$$

Dans [5], Gibbons expose un certain nombre de problèmes issus de la cosmologie faisant intervenir cette équation du type Ginzburg-Landau.

Un premier problème est de considérer le comportement asymptotique des $u_{\varepsilon}$ solutions de $-\Delta u=\frac{1}{\varepsilon} u\left(1-u^{2}\right)$ lorsque $\varepsilon$ tend vers zéro. Modica montre dans [6] que la famille des $u_{\varepsilon}$ converge lorsque $\varepsilon$ tend vers zéro vers une application $u^{*}$ qui ne prend que les valeurs 1 et -1 . De plus, la surface de séparation entre $\left\{x, u^{*}(x)=-1\right\}$ et $\left\{x, u^{*}(x)=1\right\}$ est une surface minimale. Ce passage à la limite modélise les transitions de phases dans la théorie de Van der Walls, Cahn et Hilliard.

Dans le même ordre d'idée, F. Béthuel, H. Brezis et F. Hélein étudient dans [1] le comportement des $u_{\varepsilon}$, qui sont cette fois définies sur la boule unité de $\mathbb{R}^{2}$ et à valeurs dans $\mathbb{R}^{2}$ et qui minimisent les fonctionnelles

$$
F_{\varepsilon}(u)=\frac{1}{2} \int_{B^{2}}\|\nabla u\|^{2}+\frac{1}{4 \varepsilon} \int_{B^{2}}\left(\|u\|^{2}-1\right)^{2} .
$$

Ils montrent que les $u_{\varepsilon}$ convergent vers une application $u^{*}$ qui est harmonique à valeurs dans $S^{1}$.

Dans cet article, on se limite à des applications à valeurs dans $\mathbb{R}$, et on démontre deux résultats motivés par des questions posées dans [5]. On considère les éléments de $H_{l o c}^{1}\left(\mathbb{R}^{n}\right) \cap L^{\infty}\left(\mathbb{R}^{n}\right)$ vérifiant au sens des distributions :

$$
-\Delta u+u\left(u^{2}-1\right)=0
$$

et satisfaisant les conditions à l'infini suivantes :

$$
\lim _{x_{1} \rightarrow-\infty} u=-1 \quad \text { et } \quad \lim _{x_{1} \rightarrow+\infty} u=1,
$$

ces limites étant uniformes en les autres variables $x_{2}, \ldots, x_{n}$.

Une solution est donnée par $f\left(x_{1}, \ldots, x_{n}\right)=t h\left(\frac{x_{1}}{\sqrt{2}}\right)$. Le premier résultat de cet article est le théorème suivant : 
THÉORÈmE 1. - Soit $\omega$, ouvert borné de $\mathbb{R}^{n-1}$. Sur l'ensemble $K$ des éléments de $H_{\text {loc }}^{1}\left(\mathbb{R}^{n}\right) \cap L^{\infty}\left(\mathbb{R}^{n}\right)$ vérifiant $\lim _{x_{1} \rightarrow-\infty} u=-1$ et $\lim _{x_{1} \rightarrow+\infty} u=1$, on considère la fonctionnelle $F_{\omega}$ définie par

$$
F_{\omega}(u)=\int_{\mathbb{E} \times \omega} \frac{1}{2}|\nabla u|^{2}+\frac{\left(u^{2}-1\right)^{2}}{4} .
$$

Alors, la fonction $f$ définie par

$$
f\left(x_{1}, \ldots, x_{n}\right)=\operatorname{th}\left(\frac{x_{1}}{\sqrt{2}}\right)
$$

minimise la fonctionnelle $F_{\omega}$.

Dans [5], Gibbons posait aussi le problème de l'unicité (à translation près) de la solution sur tout $\mathbb{R}^{n}$ de $(*)$ avec les conditions aux limites du théorème 1 . Ce problème reste ouvert. On montre ici un résultat d'unicité du type Liouville :

ThÉORÈmE 2. - Soit $n \geq 2$, soit $u$ un élément de $H_{l o c}^{\perp}\left(\mathbb{R}^{n}\right)$ solution sur $\mathbb{R}^{n}$, de l'équation:

$$
-\Delta u=u\left(1-u^{2}\right)
$$

telle que $u$ tend vers 1 lorsque $|x|$ tend vers $+\infty$. Alors $u$ est constante égale à 1 sur $\mathbb{R}^{n}$.

L'article est organisé comme suit. La deuxième partie est consacrée à la démonstration du théorème 1. Dans cette preuve, on étudie d'abord le problème en dimension 1 , où l'on sait montrer directement l'unicité de la solution de l'équation $(*)$ et l'existence d'un minimum de la fonctionnelle associée. Puis, en dimension quelconque, on se ramène à la dimension 1 en utilisant une transformation analogue à la symétrisation de Steiner qui décroît l'énergie et qui nous place dans le cadre des fonctions à une variable.

Dans la troisième partie, on démontre le théorème 2. Pour ce faire, on effectue, grâce au principe du maximum, une majoration asymptotique de $u$. Cette majoration permet de montrer que le gradient de $u$ est dans $L^{2}\left(\mathbb{R}^{n}\right)$ et dans un deuxième temps, de passer à la limite dans une identité de Pohozaev qui mène au résultat.

Deux problèmes évoqués dans [5] restent ouverts. D'une part, comme on l'a vu plus haut, l'unicité (à translation près) de la solution sur tout $\mathbb{R}^{n}$ de $(*)$ avec les conditions aux limites du théorème 1 . D'autre part, on sait que les $u_{\varepsilon}$ solutions de $-\Delta u=\frac{1}{\varepsilon} u\left(1-u^{2}\right)$ convergent, à extraction près vers $u^{*}$. La surface séparant $\left\{x, u^{*}(x)=-1\right\}$ et $\left\{x, u^{*}(x)=1\right\}$ étant une surface minimale, on peut chercher à construire une famille de $u_{\varepsilon}$ dont le $u^{*}$ limite est associé à une cathénoïde ou à un hélicoïde. 


\section{2. $f$ EST MINIMISANTE}

Démonstration. - Dans un premier temps, on remarque que $u$ étant bornée uniformément, on sait alors que $u$ est régulière par un argument classique de bootstrap.

\section{$1^{e r}$ pas : Problème en dimension 1}

On se place en dimension 1 . Soit $u$, élément de $H_{l o c}^{1}(\mathbb{R})$, solution de

$$
\left\{\begin{array}{l}
u^{\prime \prime}=u\left(u^{2}-1\right) \\
\lim _{x \rightarrow-\infty} u=-1 \\
\lim _{x \rightarrow+\infty} u=1
\end{array}\right.
$$

Remarque. - En dimension 1, il est inutile de supposer a priori que $u$ est uniformément bornée. En effet, par injection de Sobolev ( $c f$. [2]), u est dans un espace de Hölder $\mathcal{C}^{0, \alpha}$, et donc, grâce à un argument de bootstrap, $u$ est de classe $\mathcal{C}^{\infty}$. En raison des conditions aux limites, il est alors clair que $u$ est bornée uniformément sur $\mathbb{R}$.

Montrons que $u$ prend ses valeurs entre -1 et 1 . Supposons que $\sup _{\mathbb{R}} u=\beta>1$. En raison des conditions aux limites, il est clair que ce sup est fini et est atteint. Soit $A=u^{-1}(\{\beta\})$. Puisque $u$ est continue, $A$ est fermé. Soit $z_{0} \in A$. Le sup de $u$ est atteint en $z_{0}$, donc, $u^{\prime}\left(z_{0}\right)=0$. De plus, $\beta>1$ donc, $\beta\left(\beta^{2}-1\right)>0$ donc, $u^{\prime \prime}\left(z_{0}\right)>0$ donc il existe $I$, voisinage de $z_{0}$ tel que

$$
\forall z \in I, \quad u^{\prime \prime}(z) \geq 0
$$

Donc, $u$ est convexe sur $I$ et $u^{\prime}\left(z_{0}\right)=0$, i.e. $z_{0}$ réalise le minimum de $u$ sur $I$, or comme $z_{0}$ réalise le sup de $u$ sur $\mathbb{R}, u_{\mid I}$ est constante égale à $\beta$, i.e. $I \subset A$. Donc, $A$ est ouvert, et $\mathbb{R}$ étant connexe, $A=\mathbb{R}$, ce qui est absurde en raison des conditions aux limites.

On montre de même que $u \geq-1$ sur $\mathbb{R}$.

\section{$2^{e}$ pas : Résolution de $(*)$ en dimension 1}

On remarque que si $u$ est solution, la fonction qui à $x$ associe $u(x+\alpha)$ l'est aussi. On fixe alors la valeur de $u$ en zéro en posant $u(0)=0$. On va maintenant résoudre l'équation.

D'après le premier pas, $u^{2}-1$ reste négatif, donc $u^{\prime \prime}$ et $u$ sont de signe opposé et $u$ tendant vers -1 en $-\infty, u^{\prime \prime}$ est positif au voisinage de $-\infty$. 
Ainsi, dans un voisinage de $-\infty, u^{\prime}$ est croissante donc a une limite en $-\infty$ laquelle ne peut être que nulle puisque $u$ a aussi une limite en $-\infty$. Ainsi, $u^{\prime}$ reste strictement positif au voisinage de $-\infty$.

Soit $a$ le premier zéro de $u^{\prime}$. Montrons que $a=+\infty$, i.e. que $u^{\prime}$ ne s'annule pas sur $\mathbb{R}$. Si tel n'est pas le cas, soit $b=u(a)$. $u^{\prime}$ est strictement positive sur $]-\infty, a\left[\right.$ donc $u$ réalise un $\mathcal{C}^{1}$-difféomorphisme de $]-\infty, a[$ sur $]-1, b[$.

On peut donc prendre $u$ comme nouvelle variable et $p=u^{\prime}$ comme nouvelle inconnue. On a alors $u^{\prime \prime}=p \frac{d p}{d u}$, ainsi, $p$ est solution de

$$
\left\{\begin{array}{l}
p(-1)=p(b)=0 \\
\forall u \in]-1, b\left[, \quad p \frac{d p}{d u}=u\left(u^{2}-1\right)\right.
\end{array}\right.
$$

On intègre entre -1 et $u$ et on obtient

$$
\forall u \in]-1, b\left[, \quad p^{2}(u)=\frac{1}{2}\left(u^{2}-1\right)^{2} .\right.
$$

Or, $p(b)=0$, donc, $b=1$ et en $x=a, u(a)=1, u^{\prime}(a)=0$ donc, d'après le théorème de Cauchy Lipschitz, $u$ est constante égale à 1 , ce qui est impossible. Donc, $a=+\infty$ et on peut résoudre l'équation car on a

$$
\forall x \in \mathbb{R}, \quad u^{\prime}(x)=\frac{1}{2}\left(1-u^{2}\right) .
$$

Donc, puisque $u(0)=0$, on a

$$
\forall x \in \mathbb{R}, \quad u(x)=\operatorname{th}\left(\frac{x}{\sqrt{2}}\right)=f(x) .
$$

\section{$3^{e}$ pas : Construction d'une transformation qui décroît l'énergie}

On introduit un outil analogue à la symétrisation de Steiner (celle-ci est utilisée, par exemple, pour démontrer le théorème de Faber Krahn concernant la première valeur propre du laplacien).

Soit $\omega$, ouvert borné de $\mathbb{R}^{n-1}$. On se place sur le cylindre $C=\mathbb{R} \times \omega$. Soit u, définie sur $C$ à valeurs dans $[-1,1]$, régulière et tendant vers -1 (resp. 1) en $-\infty$ (resp. $+\infty$ ).

On définit $u^{*}$ par :

$$
\begin{gathered}
u^{*^{-1}}(0)=C \cap\left\{x_{1}=0\right\} \\
\text { si } \lambda>0, \quad u^{*^{-1}}(\lambda)=C \cap\left\{x_{1}=\frac{\operatorname{mes}(\{0 \leq u(x) \leq \lambda\})}{\operatorname{mes}(\omega)}\right\}
\end{gathered}
$$


si $\lambda<0, \quad u^{*^{-1}}(\lambda)=C \cap\left\{x_{1}=-\frac{\operatorname{mes}(\{\lambda \leq u(x) \leq 0\})}{\operatorname{mes}(\omega)}\right\}$

et ce pour $\lambda \in]-1,1[$.

Remarque 1. - La transformée de $u$, notée $u^{*}$, ne dépend que de la variable $x_{1}$ et est croissante en celle-ci.

Remarque 2. - On pose

$$
A_{u}(\mu)= \begin{cases}\operatorname{mes}\left(u^{-1}([0, \mu])\right) & \text { si } \mu \geq 0 \\ \operatorname{mes}\left(u^{-1}([\mu, 0])\right) & \text { si } \mu \leq 0\end{cases}
$$

Pour tout $\mu$, par construction de $u^{*}$, on a $A_{u}(\mu)=A_{u^{*}}(\mu)$.

On va montrer que

$$
F_{\omega}\left(u^{*}\right) \leq F_{\omega}(u)
$$

- Dans un premier temps, on montre que

$$
\int_{C}\left(1-u^{2}\right)^{2}=\int_{C}\left(1-u^{*^{2}}\right)^{2}
$$

Soit $f$, définie sur $C$ à valeurs dans $\mathbb{R}^{+}$, mesurable. Soit $\Omega_{\lambda}=\{x \in C$, $\lambda<f(x)\}$ pour $\lambda \in \mathbb{R}^{+}$. Par définition de $\Omega_{\lambda}$, on a

$$
f(x)=\int_{0}^{+\infty} 1_{\Omega_{k}}(x) d \mu
$$

Ainsi,

$$
\int_{C} f(x) d x=\int_{C} \int_{0}^{+\infty} 1_{\Omega_{\mu}}(x) d \mu d x .
$$

On peut appliquer Fubini car la fonction intégrée est positive :

$$
\int_{C} f(x) d x=\int_{0}^{+\infty} \int_{C} 1_{\Omega_{\mu}}(x) d x d \mu=\int_{0}^{+\infty} \operatorname{mes}\left(\Omega_{\mu}\right) d \mu .
$$

On applique cette formule à $f=\left(1-u^{2}\right)^{2}$ :

$$
\int_{C}\left(1-u^{2}\right)^{2}=\int_{0}^{+\infty} \operatorname{mes}\left(\left\{x,\left(1-u^{2}(x)\right)^{2} \geq \lambda\right\}\right) d \lambda
$$

Or, par construction de $u^{*}$,

$$
\begin{aligned}
\left\{x,\left(1-u^{2}\right)^{2}(x) \geq \lambda\right\} & =\{x,-\sqrt{1-\sqrt{\lambda}} \leq u(x) \leq \sqrt{1-\sqrt{\lambda}}\} \\
& =\left\{x,-\sqrt{1-\sqrt{\lambda}} \leq u^{*}(x) \leq \sqrt{1-\sqrt{\lambda}}\right\}
\end{aligned}
$$


Donc,

$$
\int_{C}\left(1-u^{2}\right)^{2}=\int_{0}^{+\infty} \operatorname{mes}\left(\left\{x,\left(1-u^{*^{2}}\right)^{2} \geq \lambda\right\}\right) d \lambda=\int_{C}\left(1-u^{*^{2}}\right)^{2} .
$$

- Montrons maintenant que

$$
\int_{C}\left|\nabla u^{*}\right|^{2} \leq \int_{C} \mid \nabla u^{2}
$$

Les deux arguments fondamentaux de la preuve sont la formule de la coaire et une égalité isopérimétrique. Cette démonstration suit de très près la preuve du résultat analogue qui concerne la symétrisation de Steiner.

On démontre l'inégalité pour $u$ régulière et un argument de densité permet de conclure.

Avec la formule de la coaire (démontrée dans [4]), si $V$ désigne l'ensemble des valeurs critiques de $u$, on a

$$
\int_{C}|\nabla u|^{2}=\int_{\mathbb{R} \backslash V}\left(\int_{u^{-1}(\mu)}|\nabla u|\right) d \mu
$$

Remarque. - Le lemme de Sard nous permet de ne pas tenir compte des valeurs critiques de $u$ qui pourraient poser problème.

On note par $L_{u}(\mu)$ la mesure de Hausdorff (n-1)-dimensionnelle de $u^{-1}(\mu)$.

On a alors, grâce à l'inégalité de Cauchy-Schwarz appliquée à $\nabla u \frac{1}{\nabla u}$ :

$$
\int_{C}|\nabla u|^{2} \geq \int_{\mathbb{R} \backslash V} \frac{\left(L_{u}(\mu)\right)^{2}}{\int_{u^{-1}(\mu)} \frac{1}{\nabla u}}
$$

De plus, toujours avec la formule de la coaire, si $0 \leq \mu_{1} \leq \mu_{2}$,

$$
A_{u}\left(\mu_{2}\right)-A_{u}\left(\mu_{1}\right)=\int_{\Omega_{\mu_{1} \backslash \Omega_{\mu_{2}}}} d x=\int_{\left[\mu_{1}, \mu_{2}\right] \backslash V}\left(\int_{u^{-1}(\mu)} \frac{1}{\nabla u} d x\right) d \mu .
$$

Donc, si $\mu \geq 0$ n'est pas valeur critique de $u$,

$$
\frac{d A_{u}}{d \mu}(\mu)=A_{u}^{\prime}(\mu)=\int_{u^{-1}(\mu)} \frac{1}{\nabla u} d x
$$

On montre la même chose pour $\mu \leq 0$. Ainsi,

$$
\int_{C}|\nabla u|^{2} \geq \int_{\mathbb{R} \backslash V} \frac{\left(L_{u}(\mu)\right)^{2}}{A_{u}^{\prime}(\mu)} d \mu
$$

Vol. $12, \mathbf{n}^{\circ} 3-1995$. 
Or, $A_{u}=A_{u^{*}}$, donc, $A_{u}^{\prime}=A_{u^{*}}^{\prime}$. De plus, par le théorème des valeurs intermédiaires, $u^{-1}(\mu)$ contient au moins une section du cylindre $C$ et la mesure (n-1)-dimensionnelle de ladite section est supérieure à la mesure de $\omega$.

Donc, $L_{u}(\mu) \geq L_{u^{*}}(\mu)$. Donc,

$$
\int_{C}|\nabla u|^{2} \geq \int_{\mathbb{R} \backslash V} \frac{\left(L_{u}(\mu)\right)^{2}}{A_{u^{*}}^{\prime}(\mu)} d \mu
$$

Or, sur $u^{*^{-1}}(\mu), \nabla u^{*}$ est constant, car $u^{*}$ ne dépend que de $x_{1}$. Donc,

$$
\int_{C}\left|\nabla u^{*}\right|^{2}=\int_{\mathbb{R}^{+}}\left(\int_{u^{*-1}(\mu)}\left|\nabla u^{*}\right| d x\right) d \mu=\int_{\mathbb{R}^{+}}\left|\nabla u^{*}\right| L_{u^{*}}(\mu) d \mu
$$

et de plus,

$$
\int_{u^{*-1}(\mu)} \frac{1}{\nabla u^{*}} d x=\frac{L_{u^{*}}(\mu)}{\nabla u^{*}}=A_{u^{*}}^{\prime}(\mu)
$$

Ainsi,

$$
\int_{C}\left|\nabla u^{*}\right|^{2}=\int_{\mathbb{R}^{+}} \frac{\left(L_{u^{*}}(\mu)\right)^{2}}{A_{u^{*}}^{\prime}(\mu)} d \mu
$$

On en déduit bien que

$$
\int_{C}|\nabla u|^{2} \geq \int_{C}\left|\nabla u^{*}\right|^{2}
$$

\section{$4^{e}$ pas : En dimension 1, $f$ est minimisante}

Pour démontrer la minimalité de $f$, puisqu'on a montré l'unicité de la solution de (1), il suffit de démontrer que le minimum est atteint, et ce sera forcément $f$.

On minimise ainsi sur $K$ la fonctionnelle $F$ avec

$$
\begin{gathered}
K=\left\{v \in H_{l o c}^{1}(\mathbb{R}, \mathbb{R}), \lim _{x \rightarrow-\infty} v=-1, \text { et } \lim _{x \rightarrow+\infty} v=1\right\} \\
F(v)=\int_{\mathbb{R}} \frac{\left|u^{\prime}\right|^{2}}{2}+\frac{1}{4}\left(u^{2}-1\right)^{2}
\end{gathered}
$$

On considère $v_{n}$, suite minimisante de $F$. Quitte à remplacer $v_{n}$ par $v_{n}^{*}$, qui sera a fortiori une suite minimisante d'éléments de $K$, on peut supposer que les $v_{n}$ sont croissantes et s'annulent en zéro. 
- On a d'abord

$$
\forall \varepsilon>0, \quad \exists A, \quad \forall n, \forall x>A, \quad 1-\varepsilon \leq v_{n}(x) \leq 1+\varepsilon .
$$

En effet, dans le cas contraire, i.e. si

$$
\exists \varepsilon>0, \quad \forall A, \exists n, \exists x_{0}>A, \quad v_{n}\left(x_{0}\right) \leq 1-\varepsilon,
$$

comme $v_{n}$ est croissante, cette inégalité serait en fait vraie pour tout $x \leq x_{0}$. Ainsi, sachant que $v_{n}(0)=0$, on a

$$
\forall x \in[0, A], \quad\left(v_{n}^{2}(x)-1\right)^{2} \geq \varepsilon^{2} .
$$

On a donc

$$
\exists \varepsilon>0, \quad \forall A, \exists n, \quad F\left(v_{n}\right) \geq \frac{A}{4} \varepsilon^{2}
$$

ce qui contredit le fait que la suite $v_{n}$ soit minimisante.

- D'autre part, soit $p \in \mathbb{N}$. Sur tout compact $[-p, p]$, on peut extraire de $v_{n}$ une sous suite $v_{\varphi_{p}(n)}$ qui converge vers $u_{p}$ dans $H^{1}$ faible, dans $L^{2}$ fort et simplement. Ainsi, par un procédé diagonal d'extraction, et par unicité de la limite faible, on peut construire une sous suite $v_{\varphi(n)}$ de $v_{n}$ et un élément $u$ de $H_{l o c}^{1}(\mathbb{R})$ tels que sur tout compact de $\mathbb{R}, v_{\varphi(n)}$ converge vers $u$ dans $H^{1}$ faible, dans $L^{2}$ fort et simplement.

- De plus,

$$
\forall \varepsilon>0, \quad \exists A, \forall n, \forall x>A, \quad 1-\varepsilon \leq v_{n}(x) \leq 1+\varepsilon,
$$

donc, $u$ tend vers 1 en $+\infty$. On montre de même que $u$ tend vers -1 en $-\infty$.

- Enfin, pour tout $A$ de $\mathbb{R}^{+}$, par des arguments de convexité de $F_{A}$, où

$$
F_{A}(v)=\int_{-A}^{A} \frac{\left|v^{\prime}\right|^{2}}{2}+\frac{1}{4}\left(v^{2}-1\right)^{2},
$$

on a

$$
F_{A}(u) \leq \lim \inf F_{A}\left(v_{\varphi(n)}\right)
$$

ainsi,

$$
F_{A}(u) \leq \lim \inf F\left(v_{\varphi(n)}\right) .
$$

Par passage à la limite lorsque $A$ tend vers $+\infty$, on en déduit donc que le minimum est atteint, et on sait alors que c'est $f$.

\section{$5^{e}$ pas : Fin de la preuve}

Montrons que $f$ est minimisante en dimension quelconque. Sur un cylindre de la forme $\mathbb{R} \times \omega$, on considère $v$ quelconque, et on remarque que $F_{\omega}\left(v^{*}\right) \leq F_{\omega}(v)$. Or $v^{*}$ ne dépend que de $x_{1}$, et on s'est donc ramené à la dimension 1 , où l'on a montré que $x \mapsto t h \frac{x}{\sqrt{2}}$ est minimum.

Donc, $F_{\omega}(f) \leq F_{\omega}\left(v^{*}\right) \leq F_{\omega}(v)$. Donc, $f$ est bien minimum de $F_{\omega}$. 


\section{PREUVE DU THÉORÈME 2}

On rappelle les hypothèses du théorème 2 . On suppose que $u \in H_{l o c}^{1}\left(\mathbb{R}^{n}\right)$ est solution de $(*)$ et que $u$ tend vers 1 lorsque $|x|$ tend vers $+\infty$. On veut montrer que $u$ est constante égale à 1 sur $\mathbb{R}^{n}$.

Un théorème analogue est montré dans [3] lorsque $u$ est définie sur $\mathbb{R}^{2}$ à valeurs dans $\mathbb{R}^{2}$ et vérifie

$$
\begin{gathered}
-\Delta u=u\left(1-|u|^{2}\right) \\
\int_{\mathbb{R}^{2}}|\nabla u|^{2}<\infty .
\end{gathered}
$$

On démontre d'abord le lemme suivant :

LEMme 1. - Soit u, élément de $H_{l o c}^{1}\left(\mathbb{R}^{n}\right)$ et vérifiant

$$
\begin{gathered}
-\Delta u+u\left(u^{2}-1\right)=0 \\
\text { et } \lim _{|x| \rightarrow+\infty} u=1 .
\end{gathered}
$$

Alors, $u$ prend ses valeurs entre -1 et 1 .

Preuve. - Montrons que $u \leq 1$. Soit $\beta>1$. On multiplie l'équation vérifiée par $u$ par $(u-\beta)^{+}$et on intègre sur la boule $B(0, R)$ de centre zéro et de rayon $R$. Puisque $u(x)$ tend vers 1 lorsque $|x|$ tend vers $+\infty$, on peut choisir $R$ tel que $u$ soit inférieur à $\beta$ sur $\partial B(0, R)$. Ainsi, sur le bord du domaine où on intègre, on a $(u-\beta)^{+}=0$. On applique alors la formule de Stokes :

$$
\int_{B(0, R)}\left|\nabla(u-\beta)^{+}\right|^{2}=\int_{B(0, R)} u\left(1-u^{2}\right)(u-\beta)^{+} .
$$

Or, le deuxième terme est négatif, car si $u<\beta$, alors $u\left(1-u^{2}\right)(u-\beta)^{+}=0$, et si $u>\beta$, on a $1-u^{2}<0$. Ainsi, on a à gauche de l'égalité un terme positif et à droite un terme négatif, donc

$$
\int_{B(0, R)}\left|\nabla(u-\beta)^{+}\right|^{2}=0,
$$

et ceci pour tout $R$ assez grand. Donc, $u \leq \beta$ et ce pour tout $\beta>1$. Donc, $u \leq 1$. On montre, en multipliant par $(u+1)^{-}$l'équation vérifiée par $u$, que $u \geq-1$. Donc, $u$ prend ses valeurs dans $[-1,1]$. 
On remarque que $u$ est uniformément bornée, donc son laplacien appartient à tous les espaces $L_{l o c}^{p}\left(\mathbb{R}^{n}\right)$. Ainsi, $u$ est dans tous les $W_{l o c}^{2, p}\left(\mathbb{R}^{n}\right)$ et ce pour $1<p<+\infty$. En appliquant une injection de Sobolev pour $p$ assez grand, on obtient que $u$ est dans un $\mathcal{C}^{1, \alpha}\left(\mathbb{R}^{n}\right)$ et par un argument de bootstrap, on montre que $u$ est régulière.

Démonstration du théorème 2.

\section{$1^{\text {er }}$ pas : Majoration asymptotique de $u$}

On pose $v=1-u$. On a $\lim _{|x| \rightarrow+\infty} v=0$ et $v \in[0,2]$. De plus, $v$ vérifie :

$$
-\Delta v+v(1-v)(2-v)=0 .
$$

La limite de $v$ lorsque $|x|$ tend vers $+\infty$ étant nulle, il existe $R$ tel que si $x$ est dans $\mathbb{R}^{n} \backslash B(0, R), 1 \leq(1-v)(2-v)$, et donc, puisque $v \geq 0$,

$$
-\Delta v+v \leq-\Delta v+v(1-v)(2-v)=0
$$

On définit sur $\mathbb{R}^{n} \backslash B(0, R): v_{0}(x)=e^{-|x|}$.

$$
\Delta v_{0}=e^{-|x|}-\frac{n-1}{|x|} e^{-|x|}
$$

Donc,

$$
-\Delta v_{0}+v_{0}=\frac{n-1}{|x|} e^{-|x|} \geq 0
$$

Donc, si on pose $w=v-t v_{0}$, on a

$$
-\Delta w+w \leq 0
$$

D'autre part, $\lim _{|x| \rightarrow+\infty} w=0$, et de plus, si $t$ est assez grand, $v-t v_{0} \leq 0$ sur $\partial B(0 . R)$, donc, avec le principe du maximum, on obtient que $w \leq 0$ sur $\mathbb{R}^{n} \backslash B(0, R)$, donc,

$$
\forall x \in \mathbb{R}^{n} \backslash B(0, R), \quad 0 \leq v(x) \leq t e^{-|x|} .
$$

Les deux pas suivants sont inspirés de la preuve du théorème 2 de [3].

\section{$2^{e}$ pas : Le gradient de $u$ est de carré intégrable sur $\mathbb{R}^{n}$}

Soit $\chi$ fonction régulière à support compact dans $\mathbb{R}^{n}$. On suppose que $\chi$ est constante égale à 1 dans $B(0,1)$, est nulle hors de $B(0,2)$ et prend ses valeurs dans $[0,1]$. On pose alors $\chi_{p}(x)=\chi\left(\frac{x}{p}\right)$ où $p \in \mathbb{N}$. 
On remarque que

$$
\Delta\left(u^{2}\right)=2|\nabla u|^{2}+2 u \Delta u
$$

et donc, puisque $u$ est solution de l'équation $(\star)$,

$$
\frac{1}{2} \Delta\left(u^{2}-1\right)=|\nabla u|^{2}+u^{2}\left(u^{2}-1\right) \text {. }
$$

On prend alors $\chi_{p}$ comme fonction test de cette équation et on obtient

$$
\frac{1}{2} \int_{\mathbb{R}^{n}}\left(u^{2}-1\right) \Delta \chi_{p}=\int_{\mathbb{R}^{n}}|\nabla u|^{2} \chi_{p}+\int_{\mathbb{R}^{n}} u^{2}\left(u^{2}-1\right) \chi_{p} .
$$

Le terme de gauche de cette égalité tend vers zéro lorsque $p$ tend vers $+\infty$. En effet,

$$
\left|\int_{\mathbb{R}^{n}}\left(u^{2}-1\right) \Delta \chi_{p}\right| \leq \frac{K}{p^{2}} \int_{B(0,2 p) \backslash B(0, p)}\left|u^{2}-1\right| .
$$

où $K$ ne dépend pas de $p$. En raison des majorations asymptotiques obtenues dans le premier pas, $\left|u^{2}-1\right|$ est dans $L^{1}\left(\mathbb{R}^{n}\right)$ et on s'aperçoit donc que le deuxième terme de cette inégalité tend vers zéro lorsque $p$ tend vers $+\infty$.

D'autre part, $u$ étant uniformément borné, $u^{2}\left(u^{2}-1\right)$ est dans $L^{1}\left(\mathbb{R}^{n}\right)$.

Enfin, en faisant tendre $p$ vers $+\infty$ dans l'égalité ci-dessus, on obtient que

$$
\int_{\mathbb{R}^{n}}|\nabla u|^{2}=\int_{\mathbb{R}^{n}} u^{2}\left(1-u^{2}\right)<\infty .
$$

Donc, $\nabla u$ est dans $L^{2}\left(\mathbb{R}^{n}\right)$.

\section{$3^{e}$ pas : Méthode de Pohozaev}

On multiplie l'équation $-\Delta u=u\left(1-u^{2}\right)$ par $\left(\sum_{i=1}^{n} x_{i} \frac{\partial u}{\partial x_{i}}\right) \chi_{p}$ et on intègre sur $\mathbb{R}^{n}$.

$$
\begin{aligned}
& -\int_{\mathbb{R}^{n}} \Delta u\left(\sum_{i=1}^{n} x_{i} \frac{\partial u}{\partial x_{i}}\right) \chi_{p}=\int_{\mathbb{R}^{n}} \nabla u . \nabla\left(\chi_{p} \sum_{i=1}^{n} x_{i} \frac{\partial u}{\partial x_{i}}\right) \\
& =\int_{\mathbb{R}^{n}}|\nabla u|^{2} \chi_{p}+\sum_{i, j} \int_{\mathbb{R}^{n}} x_{i} \frac{\partial u}{\partial x_{j}} \frac{\partial^{2} u}{\partial x_{i} \partial x_{j}} \chi_{p}+\int_{\mathbb{R}^{n}}(x, \nabla u)\left(\nabla u, \nabla \chi_{p}\right) \\
& =\int_{\mathbb{R}^{n}}|\nabla u|^{2} \chi_{p}+\int_{\mathbb{R}^{n}} \frac{x \cdot \nabla\left(|\nabla u|^{2}\right)}{2} \chi_{p}+\int_{\mathbb{R}^{n}}(x, \nabla u)\left(\nabla u, \nabla \chi_{p}\right) \\
& =\left(1-\frac{n}{2}\right) \int_{\mathbb{R}^{n}}|\nabla u|^{2} \chi_{p}-\frac{1}{2} \int_{\mathbb{R}^{n}}\left(x, \nabla \chi_{p}\right)|\nabla u|^{2} \\
& \quad+\int_{\mathbb{R}^{n}}(x, \nabla u)\left(\nabla u, \nabla \chi_{p}\right) .
\end{aligned}
$$


D'autre part,

$$
\begin{aligned}
& \int_{\mathbb{R}^{n}} u\left(1-u^{2}\right)\left(\sum_{i=1}^{n} x_{i} \frac{\partial u}{\partial x_{i}}\right) \chi_{p} \\
& =\sum_{i=1}^{n} \int_{\mathbb{R}^{n}} \frac{\partial}{\partial x_{i}}\left(-\frac{\left(1-u^{2}\right)^{2}}{4}\right) x_{i} \chi_{p} \\
& =-\sum_{i=1}^{n} \int_{\mathbb{B}^{n}}-\frac{\left(1-u^{2}\right)^{2}}{4} \chi_{p}-\sum_{i=1}^{n} \int_{\mathbb{R}^{n}}-x_{i} \frac{\left(1-u^{2}\right)^{2}}{4} \frac{\partial \chi_{p}}{\partial x_{i}} \\
& =\frac{n}{4} \int_{\mathbb{R}^{n}}\left(1-u^{2}\right)^{2} \chi_{p}+\int_{\mathbb{R}^{n}} \frac{\left(1-u^{2}\right)^{2}}{4}\left(\nabla \chi_{p}, x\right) .
\end{aligned}
$$

On fait tendre $p$ vers $+\infty$. On remarque que

$$
\left|\int_{\mathbb{R}^{n}}(x, \nabla u)\left(\nabla u, \nabla \chi_{p}\right)\right| \leq K \int_{B(0,2 p) \backslash B(0, p)}|\nabla u|^{2}
$$

où $K$ est indépendant de $p$. Or, on a montré dans le deuxième pas que le gradient de $u$ est dans $L^{2}\left(\mathbb{R}^{n}\right)$ ce qui prouve que le terme étudié tend vers zéro lorsque $p$ tend vers $+\infty$.

On montre de même que $\frac{1}{2} \int_{\mathbb{R}^{n}}\left(x, \nabla \chi_{p}\right)|\nabla u|^{2}$ tend vers zéro lorsque $p$ tend vers $+\infty$.

D'autre part, $\left(u^{2}-1\right)^{2}$ étant dans $L^{1}\left(\mathbb{R}^{n}\right)$, on obtient que $\int_{\mathbb{R}^{n}} \frac{\left(1-u^{2}\right)^{2}}{4}\left(\nabla \chi_{p}, x\right)$ tend lui aussi vers zéro quand $p$ tend vers $+\infty$.

En conclusion, le passage à la limite dans l'égalité de Pohozaev montre que

$$
\left(1-\frac{n}{2}\right) \int_{\mathbb{R}^{n}}|\nabla u|^{2}=\frac{n}{4} \int_{\mathbb{R}^{n}}\left(1-u^{2}\right)^{2}
$$

Or, $n \geq 2$, donc, $1-\frac{n}{2} \leq 0$, donc, les deux termes de l'égalité sont nuls.

Donc, $u$ est constante égale à 1 sur $\mathbb{R}^{n}$, ce qui clôt la démonstration.

\section{REMERCIEMENTS}

L'auteur est heureux d'exprimer sa gratitude à F. Hélein pour son aide et ses nombreux conseils. 


\section{RÉFÉRENCES}

[1] F. Béthuel, H. Brezis and F. Hélein, Ginzburg-Landau Vortices, Birkhaüser, 1994.

[2] H. Brezis, Analyse Fonctionnelle, Masson, 1987.

[3] H. BRezis, F. MeRle and T. RivièRe, Quantization effects for $-\Delta u=u\left(1-|u|^{2}\right)$ in $\mathbb{R}^{2}$, à paraître dans Arch. Rat. Mech. Anal.

[4] H. FEDERER, Geometric measure theory, New York, Springer, 1969.

[5] G. W. GibBons, Topological Defects in Cosmology, Private Communication.

[6] L. ModiCA, The gradient theory of phase transitions and the minimal interface criterion, Arch. Rat. Mech. Anal., Vol. 98, 1987, pp. 123-142.

(Manuscrit reçu le 28 octobre 1993; version révisée reçue le 15 février 1994.) 